\title{
Avogadro's Hypothesis after 200 Years
}

\author{
Lubomir Held
}

Department of Chemistry, Faculty of Education, Trnava University, Slovakia

Copyright $(2017$ by authors, all rights reserved. Authors agree that this article remains permanently open access under the terms of the Creative Commons Attribution License 4.0 International License

\begin{abstract}
Avogadro's conception of the structure of gases was not widely accepted by his contemporaries - probably because the hypothesis was not supported by direct evidence. This problem is rarely addressed in schools. This article discusses the difficulties that accompany the acceptance of new ideas. Such difficulties may be associated with the ways in which scientists reason. Furthermore, we have developed a simple experiment in which these complex matters can be introduced to students. Specifically, we put students into the situation that was faced by chemists 200 years ago. This article aims to discuss (1) why Avogadro's ideas were not accepted by his contemporaries, and (2) how these ideas could be used to teach chemistry in a meaningful way.
\end{abstract}

Keywords Demonstration, Avogadro's Hypothesis, Secondary Education

\section{Introduction}

For many people, a combined interest in chemistry and history is quite unusual. Indeed, I have asked colleagues from different chemical departments, "Who enjoyed history lessons at elementary or secondary school?" I have received almost no positive responses. Lessons in the history of chemistry are usually quite boring and they typically contain a lot of facts and information. Perhaps this is one reason they have been disappearing from textbooks in both elementary schools and universities.

In a number of studies, we have shown that historical or fundamental knowledge, which played a remarkably important role in the development of chemistry as a science, was part of older chemistry textbooks, and that this subject matter is completely absent from more recent or contemporary texts. Specifically, newer chemistry textbooks lack information on Dalton's figural labeling of elements, Wohler's discovery of carbamide/urea as a synthetic organic substance, and important methodologies, e.g. Cronstedt's blowpipe analysis, Hoffman's electrolyzer, or Liebig's elemental analysis.

These days, textbooks only briefly mention (as an obligatory item with no practical meaning - or as a truism) the empirical chemical laws. Moreover, some academic textbooks even fail to mention Avogadro's law-that remarkable breakthrough in the development of chemical science.

Avogadro's hypothesis (law) belongs to fundamental chemical knowledge. Recently, it has been 200 years since its publishing (1811 - 2011). Scientists had not accepted it for almost 50 years and Avogadro himself did not live long enough to see its acceptance. This moment signals that the education will struggle this as a problem too.

Avogadro's law in textbooks is being described, stated as a fact. There are visualizations of it, however there are no experiments encouraging thinking over it. Professional databases contain relatively small amount of links on demonstration of Avogadro's law. Selection of relevant works is a part of our manuscript.

How to demonstrate Avogadro's hypothesis to students? How was it possible to formulate such an insightful idea? Was it mere speculation? Could its Avogadro have had any other empirical clues?

Seeking possibilities on embedding the Avogadro's law within IBSE activities we have developed an experiment regarding this matter, which is a source of cognitive conflict for pupils (as well as adult professionals) and allows discussion on problem established by Avogadro 200 years ago. However, the experiment is very simple and easy to perform.

As suggested in the manuscript, the problem of empiric proof of Avogadro's hypothesis has methodological and philosophical potential, which lies beyond possibilities of this manuscript and requires separate theoretical study.

\section{The Fate of Avogadro's Hypothesis}

Avogadro's hypothesis, as well as its widespread acceptance, was an important breakthrough in the development of chemical science. In 1811, Avogadro [1] published his original hypothesis about the volume ratios of gases; specifically, he claimed that gases react and generate products in volume ratios of whole numbers. Avogadro related this hypothesis to the atomic ideas that had been published by Dalton a few years earlier. According to 
Avogadro's hypothesis equal volumes of all gases contain equal numbers of molecules when the gases are at the same temperature and pressure. Elaborating on this hypothesis, he asserted the volume of a gas is not dependent on the size or mass of the molecules of the gas

To explain and work further with the concept of volume ratios during chemical reactions, it was necessary to develop supplementary terms and connections. Therefore, Avogadro used the term "integral molecule". Granted, he did not know how many atoms each molecule of gas contained; however, based on the reactive ratios indicated by his hypothesis, it was possible to identify the smallest possible number. Thus, the hypothesis combined the macroscopic and microscopic views of the chemical principles. Furthermore, it detailed a very strange phenomenon; namely, that equal volumes of gas substances, under the same conditions, contain the same number of molecules. This is a theory in two parts: one about integral molecules and the other about the universal behavior of gas particles. Avogadro suggested that this phenomenon is caused by the long distances between integral molecules, which we now call elementary particles; the distance is so huge that the size of the molecules themselves is negligible. It is of fundamental physical significance that, given the same conditions, the volume of any gas is constant even though the mass of this volume, (density) is different.

In his study from 1811, cited above, Avogadro applied his ideas to infer relative atomic masses. For instance, he could determine the relative atomic mass of oxygen by calculating the density ratio of oxygen to a standard substance (e.g. hydrogen). Using this method, Avogadro concluded that oxygen had a relative mass that was very different from what was believed at that time. Today, we know that this groundbreaking discovery was aided by a lucky coincidence. In fact, Avogadro was comparing the masses of molecules and not atoms. It was a pure coincidence that both molecules later proved to be diatomic. If the atmosphere was formed mostly by ozone instead of oxygen, or if Avogadro had used helium as a standard for everything, the calculation would have been more complicated.

Now that a general equation of state has been formulated, and the Avogadro constant has been well calculated, we can link macroscopic data (gas density) with microscopic (number of particles in one gram-atom of particles). In this way, students can rely solely on their own understanding of algorithmic calculations.

It would be interesting to know on what basis Avogadro formed his hypothesis, which is now considered true - to the point where it may even have been upgraded to the status of a natural law. How was it possible to formulate such an insightful idea? Was it mere speculation? Could its author have had any other empirical clues?

Unlike Mendeleev's periodic law, which was the culmination of many years' work by earlier chemists and thus was immediately accepted, Avogadro's hypothesis was not recognized for a long time. Indeed, the community of chemists did not accept the hypothesis until almost 50 years after its publication, when a contribution called "A draft of a chemical philosophy course", which had been published in 1858, was presented by Stanislao Cannizzaro [2] at the Chemical Congress in 1860.

\section{Avogadro's Hypothesis and Methodological Issues}

The rejection and final acceptance of Avogadro's hypothesis indicate that many of the contentious issues mentioned above were caused by methodological differences. Be that as it may, few authors have studied this subject area. There has been an explosion of publications regarding new compounds, new properties, and the behavior of substances. However, the literature is sparse regarding the origin and nature of Avogadro's hypothesis because it falls within the scope of only a few publications focusing on history, epistemology, logic and philosophy, and perhaps sociology. Nevertheless, we shall have a look at some previous arguments in this field.

Biographical and historical studies have attributed the initial failure of Avogadro's hypothesis to various "sociological" facts. For example, K. J. Leidner [3] discussed Avogadro's bad French and a number of irrelevant details in his most important publication. Many sources replicate this view. Furthermore, J. H. Brooke [4], in his philosophical case study, indicated that the following issues were also pertinent:

- Avogadro's was geographically isolated, had an aversion to travel, and failed to sustain private scientific correspondence;

- his publication channels were inadequate, especially in the case of his later papers;

- his research program reduced chemistry to the quantification of physical forces, rendering his theories inaccessible and possibly irrelevant to the mainstream development of chemistry;

- the Berthollet-Laplace program, which underpinned his work, was itself about to crumble;

- serious problems of comprehension arose whenever attempts were made to span the chemical-atomic and physical-molecular frameworks;

- Avogadro's divisible molecules were designed ad hoc simply to save his equal numbers hypothesis;

- it was not Avogadro's intention to determine atomic weights: his ontology was such that he did not systematically pursue the question of sub-molecularity in the elementary gases;

- his extension of the equal numbers hypothesis to the solid elements looked contrived;

- the equal numbers hypothesis was of limited application;

- vapor density data were sometimes intractable and anomalous;

- Chemists could proceed, as Berzelius did, with recourse to even simpler generalizations (e.g. equal volumes of elementary gases contain equal numbers of atoms);

for Berzelius and those who adopted his


electrochemical theory, polyatomic elementary molecules were inconceivable;

- to draw analogies between organic and inorganic compounds and thus extend Berzelius's dualistic rubric into organic chemistry, the freedom to multiply or divide formulae was necessary;

- when chemists like Ampere, Gaudin, Baudrimont, and Laurent explored the theoretical possibilities of divisible elementary molecules, they focused on either crystallographic analogies, which constituted apparently premature attempts to probe intra-molecular structure, or on other, broader objectives, which were generally considered suspect;

- in his final analysis, Avogadro's hypothesis was unverifiable;

- until the work of Clausius and Maxwell had been published, the hypothesis itself was not deducible from an independent physical theory;

- during the 1830 s and ' 40 s, when dualistic analogies between organic and inorganic compounds had been well-established, accepting the molecular weights consistent with Avogadro's hypothesis would have destroyed a whole range of inter-locking conceptual networks.

Brooke went on to ask the following: "Is it actually possible that these reasons alone decided how scientific hypotheses were to be accepted?"

In addition to the sociological reasons mentioned above, the professional literature makes other speculations regarding the rejection of Avogadro's hypothesis. Furthermore, in the many years since its formulation, Avogadro's hypothesis has not been satisfactorily explained because it is not based on any direct evidence, and it cannot be verified in a positivistic sense; instead, Avogadro's law may constitute "fundamental knowledge", rather like a mathematical postulate (axiom), which cannot be verified, but is assumed to be true. Perhaps this explains Mayer's [3] declaration in 1864 regarding the congress in Karlsruhe: "It was as though scales fell from my eyes, doubt vanished, and was replaced by a feeling of peaceful certainty".

Another study discussing why scientists of the time rejected Avogadro's hypothesis focuses on logic. A. Drago and R. Oliva [5] argued that the formal logical concept of double negation may have caused chemists to misunderstand the hypothesis. In their introduction, these authors proposed that this logical process was commonly used in 19th century scientific works. Using historical scientific texts (mainly in the area of physics), they argued that Avogadro implemented double negation, or proof by conflict, which is used in the area of mathematics. The main focus of their work was getting to the core of Avogadro's idea. They showed that Avogadro, in an attempt to support his idea, pointed to evidence that was based on double negation. Drago and Oliva concluded that the final acceptance of Avogadro's ideas overcame traditional logic.

On a different note, G. Gorin [6] highlighted the uncertainty that accompanies categorizing Avogadro's ideas. Specifically, he posed the following question in the title of his lecture on the history of chemistry at the American Chemical Society conference: “Avogadro's idea: Is it hypothesis, theory, law, principle what-have-you?" Certainly, when the idea was published (1811), it was only a hypothesis; it was for this reason that it was rejected by the relevant authorities of chemical research (e.g. Dalton, Berzelius, etc.). Gorin supposed that only after 1850 did molecular-kinetic theory replace caloric theory. That is, it was accepted after all. In the modern literature, Avogadro's idea is often presented as a law, even though this description is not always applicable. In particular, it only applies in cases of relatively low pressure - not in instances of relatively high pressure, at the point where gases liquefy. For this reason, Gorin presented Avogadro's theory as a principle, rather than a law. Perhaps we could reformulate the hypothesis to mention that it only applies when the sizes of the molecules in a gas, with respect to their common distances, are almost negligible. Thus, Avogadro's principle draws on a chemical theory that is similar to the axioms of Euclid's geometry, although not as distinct.

Gorin considers the following analogy: mathematical axioms are assumed rather than proved; Avogadro's hypothesis has a similar, though less tangible, role in chemical theory: it cannot be verified using inductive reasoning.

A work by R. L. Causey [7] takes us further in the direction of the philosophy of natural science. He stated that Avogadro's hypothesis and many other theories in early chemical science are connected with the Duhemian problem or Duhem-Quin thesis. The problem — pointed out by Pierre Duhem - may have caused Avogadro's hypothesis to be rejected by contemporary chemists.

\section{Demonstration and Materials}

Prompted by the issues mentioned above, we have tried to find empirical demonstrations that would provide convincing evidence for pupils that Avogadro's hypothesis connects the macroworld (volume of gas) with the microworld (number of particles). However, after years searching the literature and internet, we have not found any such suitable empirical evidence. That is, the internet and professional literature does contain a number of presentations explaining Avogadro's hypothesis. However, these constitute a mere interpretation and clarification of the terms used, for example Bouma [8], Young [9]. Ultimately, it is necessary to carry out experiments that do not contradict this hypothesis. Several companies that produce tools based on Avogadro's hypothesis sell a complicated apparatus that allows the user to observe volume proportions during the synthesis of water from its original elements. However, this experiment "merely" demonstrates the principles discovered by Gay-Lussac.

It is common scenario recommended by many 
publications and web sites to show two balloons of the same size containing different gases and ask the pupils question which balloon contains more gas particles. It is very difficult for them to answer the question.

Thus, we have made a great effort to create a suitable presentation of Avogadro's hypothesis for use in schools. The results of this effort were presented in a conference (Held [10]).

To perform the demonstration, we needed a 50-ml syringe, weights of approximately two kilograms, a syringe cap with a piece of hose and a clamp, gas samples (e.g. helium for children balloons, carbon dioxide, methane in the form of natural gas, butane lighter fluid, air).

The procedure is as follows: pupils (or students) are given three syringes (one filled with salt, the second with water, and the third with air). They are asked to compress the syringes and are subsequently convinced that gases, in contrast with solids and liquids, compressible. This part of the demonstration is inspired by B. Criswell [11].

Next, the following experiment is prepared: the two-kilogram weight is placed on the plunger of the syringe containing gas, f.i. helium. The students are presented with the problem and asked to make an assumption: how will the plunger position of the syringe, and thus the volume of the gas change with different gas of different density and different mass of particles (f.i. butane or carbon dioxide, etc.) when the same pressure is applied? (Figure 1)

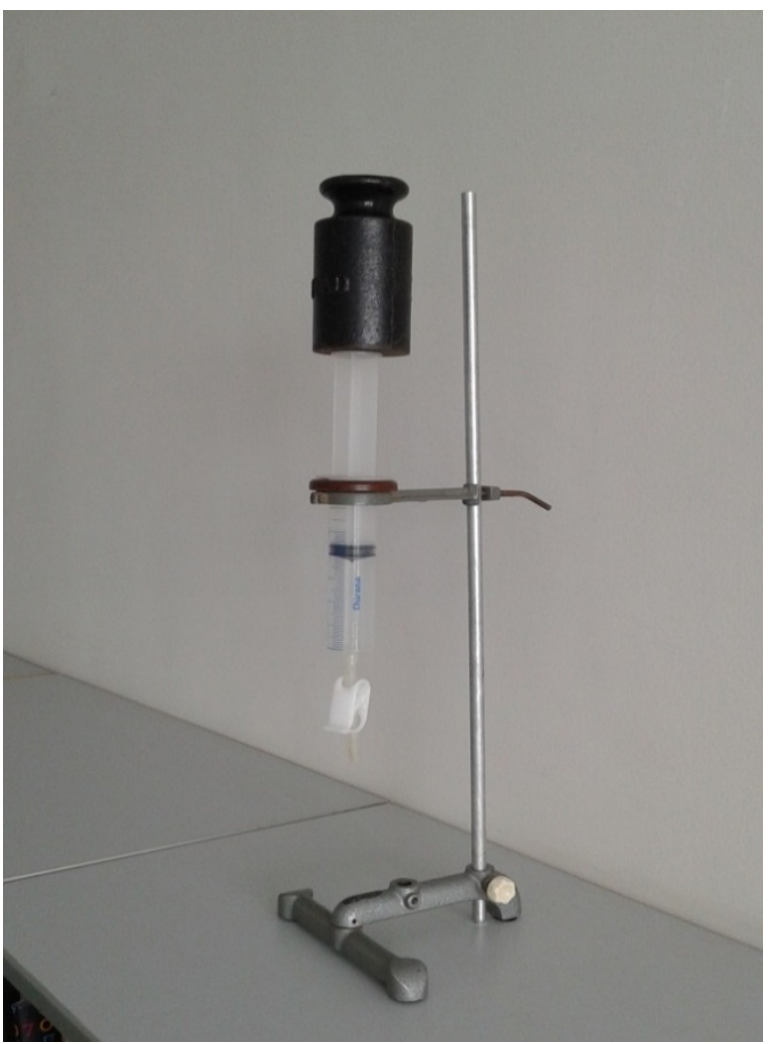

Figure 1. Apparatus for demonstrating Avogadro's hypothesis.

The majority (or all) of students of chemical education (as well as university teachers) anticipate a change of volume that depends on the relative molecular mass of the gas particles. This assumption is in fact contradicted by the Avogadro's law; however, it is intuitive for most people. In the next step we change the gas in the syringe and thus we demonstrate that it is not true: the volume change of all available gases, at the same pressure and temperature, does not differ. This moment allows students to consider the long distances between particles; the distance is so huge that the size of the molecules themselves is negligible.

\section{Conclusions}

In the matter of chemistry education, didacticians are quite unanimous: they adopt the ideas clearly formulated by A. H. Johnson [12]. Chemistry is objectively difficult because it is represented and described in three ways that are independent, but mutually interlinked: macroscopic, microscopic, and symbolic. The perception of chemical phenomena through each of these lenses constitutes advanced chemical thinking. Thinking in such a way is typical for scientists and experts in the subject of chemistry. However, linking these three worlds causes big problems for pupils and students. In addition, it leads to various misconceptions in pupils, as well as in the adult, non-specialist public, as indicated by the hundreds of didactic investigations on this subject that have been published in the last 30 years.

Within this context, we can conclude that more detailed didactic/instructional work with Avogadro's hypothesis at schools would constitute good instructional material for developing connections between the chemical micro- and macro-worlds.

The problem of acceptance in science illustrates a didactical problem: problem situations can themselves provide a cognitive tension that can be used didactically. Because of all the points mentioned above, it became taboo to empirically demonstrate Avogadro's hypothesis in education. For this reason, it is important to give pupils a strong basis that allows them to link their macroscopic and microscopic thinking as it regards chemical problem-solving. This approach would include the most problematic, but at the same time fundamental, moments in the history of chemistry.

Our experience shows that our school demonstrations are interesting and unusual; they make work with gases more accessible and use simple aids. The results are surprising for pupils and evoke a cognitive conflict that is a good starting point for further education regarding specialist terms, development of research competencies, and contact with the abstract ideas of science methodology. We contend that historic moments in chemistry are not appreciated in education, despite their big potential. One good example of this is the problem and fate of Avogadro's hypothesis.

We assume that our theoretical study leading to practical presentation of original demonstration will continue by creating IBSE activities for pupils with following education research.

As we suggested in theoretical part, there are many epistemological problems about Avogadro's hypothesis. We 
believe that this material has a potential to draft some epistemological problems to students of chemical education.

\section{Acknowledgements}

This work was supported by Slovak Research and Development Agency under the contract No. APVV-14-0070.

\section{REFERENCES}

[1] Avogadro, A. Essay on a Manner of Determining the Relative Masses of the Elementary Molecules of Bodies, and the Proportions in Which They Enter Into These Compounds. Journal de physique, 73: 58-76 (1811). Translation from Alembic Club Reprints, No. 4, "Foundations of the Molecular Theory: Comprising Papers and Extracts by John Dalton, Joseph Louis Gay-Lussac, and Amadeo Avogadro, (1808-1811) 20. 5. 2016.

http://www.chemteam.info/Chem-History/Avogadro.html. (Accessed May 5, 2016).

[2] Cannizzaro, S. Sketch of a course of chemical philosophy. Alembic Club. University of Chicago Press: Chicago, 1911, No. 18,72 p.

https://ia801407.us.archive.org/29/items/sketchofcourseof00c annrich/sketchofcourseof00cannrich.pdf (accessed May 5, 2016).

[3] Laidler, K. J. The World of Physical Chemistry. Oxford University Press: New York, 1993, p 134.
[4] Brooke, J. H. Avogadro's Hypothesis and its Fate: A Case-study in the Failure of Case-studies. History of Science. 1981, pp. 235-273.

[5] Drago, A., Oliva, R. Atomism and the Reasoning by a Non-Classical Logic. HYLE - International Journal for Philosophy of Chemistry, [Online] 1999, 5, 43-55. (Accessed May 5, 2016).

[6] Gorin, G. Avogadro's idea: It is hypothesis, theory, law, principle, what-have-you? In Abstracts of papers of the American chemical society. April 13, 1997. Vol 2, hist. part 1, p. 213.

[7] Causey, R. C. Avogadro's Hypothesis and the Duhemian Pitfall. Journal of Chemical Education. 1971, 48, 365-367.

[8] Bouma, J. Gas cans and gas cubes: Visualizing Avogadro's Law. Journal of Chemical Education, 1986, 63, p 586-587.

[9] Young, J. A., Plumb, R.C. Demonstrating Avogadro's hypothesis with the molecular dynamics simulator. Journal of Chemical Education, 1972, 49, 709-710.

[10] Held, L. Avogadro's Hypothesis as a Critical Moment in Inductive Approach How to Build a Concept of Particle Composition of Gases. In DidSci 2016 Procedings o the 7th International Conference on Research Didactics of the Science. Jun 29 - July 1, 2016. Ed: Ciesla, P. Pedagogical University of Cracow - Institute of Biology - Department of Education of Natural Sciences: Kraków, p. 47-49. [Online] http://ukftp.truni.sk/epc/13438.pdf

[11] Criswell, B. Teaching Avogadro's Hypothesis and Helping Students To See the World Differently. Journal of Chemical Education. 2008, 85, 1372-1376.

[12] Johnstone, A. H.: Teaching of Chemistry - Logical or Psychological? Chemical Education: Research and Practice in Europe, 2000, (1), pp. 9-15. 